\title{
THE DARK MATTER AND ENERGY IN THE DE SITTER WORLD
}

\author{
Bala Ali RAJABOV* \\ N.Tusi Shamakhi Astrophysics Observatory, National Academy of Sciences of Azerbaijan, Baku, Azerbaijan
}

\begin{abstract}
It is shown that dark matter and energy are cosmological quantum effects. De Sitter world is considered as a cosmological model. It is shown that in the de Sitter world, gravity and anti-gravity are different states of the Wigner elementary quantum system. It has been proven that in the Minkowski world, which is the limiting case of the de Sitter world, antigravity can be excluded. Moreover, it is shown that the Wigner - Inönü limit of the de Sitter model to the Minkowski world plays the role of Bohr's correspondence principle in quantum mechanics. A plan for further research is proposed.
\end{abstract}

Keywords: de Sitter world, Wigner-Inönü limit, Dark matter and energy, Wigner elementary systems, Correspondence principle

\section{INTRODUCTION}

Currently, the phenomena of dark matter and energy are the main problems of cosmology, and the study of their nature and constituent particles is one of the actual problems of high-energy physics, $[1,2]$.

It should be noted that the dark matter and energy are not the only problem connecting cosmology with high-energy physics. There are other fundamental problems of theoretical physics whose developmental history shows that their solution can be found at the intersection of elementary particle physics and cosmology. Particle theory is based on quantum field theory, while cosmology is based on Einstein's general theory of relativity.

Quantum field theory was created because of the unification of quantum mechanics and special relativity, the highest achievement of which is the unified theory of electroweak interactions (WeinbergSalam-Glashow theory) and quantum chromodynamics. Quantum field theory is the only conceptually and mathematically developed theory successfully used in high-energy physics, which, unlike other well-known speculative theories, is fully consistent with experimental data, [3].

But, despite these achievements, quantum field theory has serious problems, the most famous of which is the appearance of infinite values of such fundamental quantities as mass and charge. Although using various renormalization procedures one can obtain numerical results that coincide with high precision with experimental data, it is impossible to exclude infinite values, which makes the theory mathematically and physically imperfect.

Another theory successfully applied in cosmology and confirmed by numerous observations is Einstein's general theory of relativity, $[4,5,6]$. Nevertheless, despite numerous attempts, it is still impossible to combine the general theory of relativity with quantum mechanics and build a quantum theory of gravity. The general theory of relativity without quantization also has its difficulties, for example, the problems of singularities, the laws of conservation of energy and momentum, the compatibility of solutions of the Einstein equations and the basic principles of the theory, and others, [7, 8].

*Corresponding Author: balaali.rajabov@mail.ru

Received: $30.10 .2020 \quad$ Published: 27.11.2020 
However, a comparison of the results of quantum field theory and general relativity shows that only a synthesis of the concepts of both theories will help find a solution to these difficulties.

As an example, consider the problem of electric charge. From the classical works of Wigner, it is known that the spin and mass of an elementary particle have a quantum and relativistic nature. Wigner proved that spin and mass are invariants of projective representations of the Poincarè group, [9].

Nevertheless, the nature of the electric charge has not yet been clarified. On the other hand, it is in the general theory of relativity that Einstein's equations have solutions (the so-called Schwarzschild and Kerr solutions), which describe black holes with mass, angular momentum, and electric charge, [10]. Moreover, the mass, angular momentum, and electric charge of the black hole appear as integration constants for the solutions of the Einstein equations and completely define the black hole as a cosmological object, regardless of the black hole's prehistory, [11, 12].

Attempts to generalize the quantum field theory in the Minkowski world to the quantum field theory in the curved space-time of general relativity have not been successful.

In this article, we show that dark matter and energy, regardless of their constituent particles, correspond to different states of a quantum system, and this phenomenon is essentially a cosmological quantum effect. We choose de Sitter's world as a cosmological model, since it firstly, like the world of Minkowski, possesses the maximum group of movements, and secondly, in the limit, when the radius of curvature of space-time becomes infinite, it goes into the flat world of Minkowski.

This circumstance makes it possible to use the operation of contraction of the de Sitter group into the Poincarè group. In their paper, Wigner and Inönü proposed a contraction operation on the transition from the Poincarè group to the Galileo group, where the speed of light plays the role of the contraction parameter. [13]. Subsequently, the contraction operation has generalized to a wide class of Lie groups and Lie algebras by many authors, [14].

In addition, we show that in this case, the role of a contraction operation is analogous to the role of the Bohr correspondence principle. Before creating the Heisenberg matrix mechanics and Schrodinger wave mechanics, N. Bohr successfully applied the correspondence principle to study the quantum nature of atomic phenomena of a certain class, $[15,16,17]$. In our work, the operation of contraction replaces the principle of the correspondence of Bohr, since we do not have a complete theory of quantum gravity.

\section{WIGNER THEORY OF ELEMENTARY SYSTEMS}

A real understanding of the nature of the spin and mass of elementary particles appeared after the classical work of E.Wigner on the representations of the inhomogeneous Lorentz group (that is, the Poincarè group, 1937), [9]. He established that the projective representations of this group act in the Hilbert state space and that these representations are equivalent to vector representations of the universally covering group of the Poincaré group. He introduced the concept of an elementary system and showed that such systems correspond to irreducible representations, that is, the space of these representations has no invariant subspaces. Moreover, Wigner proved that the rest mass and the spin of an elementary particle are invariants that uniquely characterize irreducible representations of the Poincarè group.

In Einstein's theory of special relativity, the Poincarè group $P_{+}^{\uparrow}$ plays the role of the group of space-time symmetries. And in relativistic quantum mechanics instead of the Poincare group, the universal covering group of the Poincarè group $P_{+}^{\uparrow}$ (so-called quantum mechanical Poincarè group, $\overline{P_{+}^{\uparrow}}$ ) plays the role group of the symmetry because the states of quantum mechanical systems form the space rays, $[14,18]$. On the other hand, the unitary ray representations of the Poincarè group, i.e. projective representations are 
equivalent to unitary irreducible representations of the quantum-mechanical Poincarè group. It is for this reason that the unitary irreducible representations (UIR) of the quantum-mechanical Poincarè group $\overline{P_{+}^{\uparrow}}$ describe the state of elementary particles. Invariants of the Poincarè group $\overline{P_{+}^{\uparrow}}$ (mass and spin) characterize the invariant properties of elementary particles, and the generators of the Poincarè group determine the set of observables that determine the states of elementary particles during independent measurements.

Invariants of the inhomogeneous Poincarè group (Casimir operators) are constructed using translation generators $P_{\mu}$ and 4-dimensional rotations $M_{\mu \nu}$ as follows, [19]:

$$
m^{2}=P_{\mu} P^{\mu} ; \quad w^{2}=w_{\mu} w^{\mu}=m^{2} s(s+1) ; \quad w_{\varrho}=\epsilon_{\lambda \mu \nu \varrho} P^{\lambda} M^{\mu \nu}
$$

Here,

$m, s$ - mass and spin of the particle, respectively, and $w_{\varrho}$ is the Pauli-Lubansky-Bargmann vector.

Important! In the case $m^{2}>2$, there is a third invariant - the sign of energy:

$$
\varepsilon=\frac{P_{0}}{\left|P_{0}\right|}= \pm 1
$$

\section{EINSTEIN's EQUATIONS AND DE SITTER's SOLUTIONS}

According to Einstein's general relativity, the metric properties of space-time are determined by the distribution and motion of matter, $[5,6]$ :

$$
R_{\mu \nu}-\frac{1}{2} g_{\mu \nu} R_{\lambda}^{\lambda}+\Lambda g_{\mu \nu}=-\frac{8 \pi G}{c^{4}} T_{\mu \nu}
$$

Here,

$R_{\mu \nu}$ - the Ricci tensor, $g_{\mu \nu}$ - the metric tensor, $\Lambda$ - the cosmological constant, $G-$ Newton's gravitational constant, $T_{\mu \nu}-$ the energy-momentum tensor and $\lambda, \mu, v=0,1,2,3$.

This equation can be rewritten in an equivalent form:

$$
R_{\mu \nu}=\Lambda g_{\mu \nu}-\frac{8 \pi G}{c^{4}}\left(T_{\mu \nu}-\frac{1}{2} g_{\mu \nu} T_{\lambda}^{\lambda}\right)
$$

It is clear from (2) that the $\Lambda-$ term, even in the absence of matter $\left(T_{\mu \nu}=0\right)$, changes the spacetime geometry and $\Lambda g_{\mu \nu}$ is the energy-momentum tensor of the vacuum. ${ }^{1}$

In the vacuum, the Einstein equations take the form:

$$
R_{\mu \nu}=\Lambda g_{\mu v}
$$

\footnotetext{
${ }^{1}$ The values of $G$ and $\Lambda$ are constantly refined with the accumulation of observations:

$$
G=6.6743 \cdot 10^{-8} \frac{\mathrm{sm}^{3}}{g \cdot \mathrm{sec}^{2}}, \quad(2020) ; \quad \Lambda \sim 1.1056 \cdot 10^{-56} \mathrm{sm}^{2}, \quad \text { (2018). }
$$
}


For $\Lambda=0$, the solution of (4) is the Minkowski manifold with a group of Poincarè motions.

In the general case, the solutions of the Einstein equations (1)-(2) do not have a group of motions. However, in 1917 Willem de Sitter found two solutions of (4) for $\Lambda \neq 0$ with different global groups of movements, $[4,8]$ :

$$
\begin{aligned}
& d s^{2}=\frac{d r^{2}}{1-\frac{r^{2}}{R^{2}}}+r^{2}\left(d \vartheta^{2}+\sin ^{2} \vartheta d \varphi^{2}\right)-\left(1-\frac{r^{2}}{R^{2}}\right) c^{2} d t^{2}, \quad \text { if } \quad \Lambda>0 \\
& d s^{2}=\frac{d r^{2}}{1+\frac{r^{2}}{R^{2}}}+r^{2}\left(d \vartheta^{2}+\sin ^{2} \vartheta d \varphi^{2}\right)-\left(1+\frac{r^{2}}{R^{2}}\right) c^{2} d t^{2}, \quad \text { if } \quad \Lambda<0
\end{aligned}
$$

Here the radius $R$ of the space and the cosmological constant $\Lambda$ are related by the following formula: ${ }^{2}$

Using stereographic projections:

$$
\Lambda= \pm \frac{3}{R^{2}}
$$

$$
\xi_{1}=r \cos \vartheta ; \quad \xi_{2}=r \sin \vartheta \cos \varphi ; \quad \xi_{3}=r \sin \vartheta \sin \varphi ;
$$

and

$$
\xi_{4}=R \sqrt{1-\frac{r^{2}}{R^{2}}} \cosh \left(\frac{c t}{R}\right) ; \quad \xi_{0}=R \sqrt{1-\frac{r^{2}}{R^{2}}} \sinh \left(\frac{c t}{R}\right) .
$$

$$
\eta_{1}=r \cos \vartheta ; \quad \eta_{2}=r \sin \vartheta \cos \varphi ; \quad \eta_{3}=r \sin \vartheta \sin \varphi ;
$$

$$
\eta_{4}=R \sqrt{1+\frac{r^{2}}{R^{2}}} \cos \left(\frac{c t}{R}\right) ; \quad \eta_{5}=R \sqrt{1+\frac{r^{2}}{R^{2}}} \sin \left(\frac{c t}{R}\right) .
$$

de Sitter solutions can be isometrically embedded as sub-manifolds in 5-dimensional pseudo-Euclidean spaces:

and

$$
\xi_{0}^{2}-\xi_{1}^{2}-\xi_{2}^{2}-\xi_{3}^{2}-\xi_{4}^{2}=-R^{2}
$$

respectively.

$$
\eta_{1}^{2}+\eta_{2}^{2}+\eta_{3}^{2}-\eta_{4}^{2}-\eta_{5}^{2}=-R^{2}
$$

These spaces have global symmetry groups $S O(4,1)$ and $S O(3,2)$ that leave the metrics (7)-(8) invariant. Groups $S O(4,1)$ and $S O(3,2)$ are called de Sitter groups. Spaces $(5),(7)$ and $(6),(8)$ are called de Sitter worlds of the 1st and 2nd kind or according to the modern terminology, de Sitter worlds dS and anti de Sitter AdS, respectively.

We restrict ourselves to the de Sitter world (5), (7), and the $S O(4,1)$ group. We will consider the case of the anti-de Sitter world in a separate article because of the difficulties in interpreting space-time measurements.

The commutation relations for the generators of the group $S O(4,1)$ have the form:

\footnotetext{
${ }^{2}$ It is easy to see that for $\Lambda \rightarrow 0$, i.e. $R \rightarrow \infty$, both solutions (4)-(5) are transferred to the flat world of Minkowski.
} 


$$
\begin{gathered}
{\left[M_{\mu v}, M_{\varrho \sigma}\right]=i\left(g_{\mu \varrho} M_{v \sigma}-g_{\mu \sigma} M_{v \varrho}-g_{v \varrho} M_{\mu \sigma}+g_{v \sigma} M_{\mu \varrho}\right)} \\
{\left[M_{\mu v}, P_{\varrho}\right]=i\left(g_{\mu \varrho} P_{v}-g_{v \varrho} P_{\mu}\right) ; \quad\left[P_{\mu}, P_{v}\right]=\frac{i}{R^{2}} M_{\mu v}}
\end{gathered}
$$

where

$$
P_{\mu}=\frac{1}{R} M_{4 \mu}
$$

The Casimir operators of the Lie algebra of the group $S O(4,1)$ have the following form, [19]:

and

$$
C_{1}=-\frac{2}{R^{2}} M_{a b} M^{a b}=-P_{\lambda} P^{\lambda}-\frac{1}{R^{2}} M_{\mu \nu} M^{\mu \nu}=M^{2},
$$

$$
C_{2}=W_{a} W^{a} ; \quad W_{a}=\frac{1}{8 R} \varepsilon_{a b c d e} M^{b c} M^{d e} .
$$

To consider the result of the contraction $R \rightarrow \infty$, it is convenient to represent the operator $C_{2}$ in the following form:

where

$$
C_{2}=-V_{\lambda} v^{\lambda}-\frac{1}{R^{2}} V_{4}^{2}
$$

$$
\begin{aligned}
& V_{\lambda}=-\frac{1}{2} \varepsilon_{\lambda \varrho \mu \nu 4} P^{\varrho} M^{\mu \nu}, \\
& V_{4}=\frac{1}{8} \epsilon_{\lambda \mu \nu \varrho} M^{\lambda \mu} M^{v \varrho} .
\end{aligned}
$$

From the last expressions, it is clear that when $R \rightarrow \infty$ the Lie algebra of the group $S O(4,1)$ becomes over to the Lie algebra of the Poincare group, and the Casimir operators become:

$$
\begin{aligned}
& C_{1} \rightarrow-P_{\lambda} P^{\lambda}=m^{2}, \\
& C_{2} \rightarrow-V_{\lambda} v^{\lambda}=m^{2} s(s+1) .
\end{aligned}
$$

where $s, m$ are spin and rest mass, respectively. ${ }^{3}$

From the limiting transition of the Casimir operators $C_{1}, C_{2}$, it follows that unlike the Minkowski world in the de Sitter world, elementary systems are identified not by mass and spin, but by some functions of spin and mass.

Important! It is obvious that any function of invariants is invariant. Therefore, as invariants characterizing the unitary irreducible representations of the group $S O(4,1)$, any pair of functionally independent invariants can be chosen to identify the unitary irreducible representations of the group $S O(4,1)$.

In particular, non-degenerate representations of the group $S O(4,1)$ can be constructed in the space of $(2 s$ +1 )-component vector-functions and the degree of homogeneity of $\sigma$ on the upper field of the cone, [15]. Then the parameters $s$ and $\sigma$ will play the role of invariants characterizing the irreducible representations of the group $S O(4,1)$.

\footnotetext{
${ }^{3}$ This limiting transition corresponds to the well-known Wigner- Inönü contraction, [13, 14, 19].
} 
In addition, as will be shown in the next section, after the operation of contraction, the parameter $s$ becomes into spin and $\sigma$ to the function of mass $m$.

The basis for our assumption about the nature of dark matter and energy is the following theorem on the contraction of representations of the de Sitter group $S O(4,1)$ to the representations of the Poincarè group $\operatorname{ISO}(3,1)$.

Theorem. The result of the contraction of the UIR's, $T^{(\sigma, s)}(g) ; g \in S O(4,1) ; \sigma=-3 / 2+i m R$, for $R \rightarrow \infty$ is the direct sum of UIR, $U^{(m, s ; \varepsilon)}(g) ; g \in I S O(3,1)$, with mass $m$, spin $s$ and differing in energy sign $\varepsilon$.

The proof of the theorem is given in the article $[20,21]$.

\section{CONCLUSION}

In the flat world of Minkowski, Wigner's elementary systems are determined by the rest mass, the spin and the sign of their energy can be identified with elementary particles. The requirement of stability of physical systems forces us to limit the energy spectrum from below and exclude negative energies.

In the de Sitter world, elementary Wigner systems are identified by spin and by a parameter, which is the flat limit of a function of spin and mass, with different energy signs. However, unlike the Minkowski world, we can not exclude negative energies from consideration.

Thus, elementary systems on a cosmological scale can be in states with positive and negative energies. Elementary systems in a state with positive energy behave like a gravitating mass, and in a negative energy state as an anti-gravity mass.

We arrive at the following conclusions:

1. Dark matter and energy consist of such elementary systems;

2. Dark matter and energy are the first manifestations of quantum properties on the scale of the universe. Until now, quantum phenomena have been encountered in the micro-world, and also as macroscopic quantum effects in the theory of condensed matter;

3. Dark matter and energy are carriers of information about the first moments of the universe after the Big Bang.

The last conclusion follows from the fact that according to the standard cosmological model, the de Sitter world is a necessary phase of the evolution of the universe in the first instants $\left(10^{-34}-10^{-32}\right.$ seconds) after the Big Bang, (see reviews on cosmology in [22, 23]).

In our opinion, sterile neutrinos are the most suitable for the role of particles that make up dark matter, because they are massive, and they participate only in gravitational interactions. It follows from the above analysis that in the de Sitter's world, such particles can be in two quantum states with positive or negative energies, i.e., be gravitational or anti-gravitational.

Of course, our universe is not the de Sitter's world, although according to some data it is developing in the direction of this model, [22]. Considering the given phenomena of dark matter and dark energy in the general case is a difficult task because today there is no quantum theory of gravity. The solution of this problem in general for the gravitational field requires not only new physical concepts but also new mathematics.

From this point of view, it is very interesting to recall the history of the origin of quantum mechanics. Even before the creation of Schrodinger wave mechanics and Heisenberg matrix mechanics, N.Bohr introduced the concept of stationary orbits (the so-called Bohr orbits) and the correspondence principle 
to study atomic phenomena, $[15,17]$. The last principle allowed the use of the concepts of classical mechanics to interpret the experimental data of atomic physics before the creation of complete quantum mechanics. In our case, the operation of contraction plays the role of the Bohr principle of correspondence in the absence of the quantum theory of gravity and allows us to use the concepts of mass and spin from the at Minkowski world in the world of de Sitter. A common property of the worlds of Minkowski and de Sitter is that both worlds possess the maximum group of movements (the Poincarè $I S O(3,1)$ and de Sitter $S O(4,1)$ groups, respectively). This circumstance allows us to use the contraction operation and the well-known results of relativistic quantum field theory, [3].

But, in the general case, a solution to the problem of quantizing the gravitational field is required. This problem cannot be solved in the same way as an electromagnetic field or a general gauge field. The first main problem is that the general theory of relativity postulates a fundamental experimental fact about the equality of gravitational and inertial mass. According to the well-known work of Bohr-Rosenfeld, [24], precisely due to a decrease in the ratio of the charge of a test particle to its mass, it is possible without contradicting the interpretation of quantum measurements in quantum electrodynamics.

The analysis carried out by Bohr-Rosenfeld to measure the quantized electromagnetic field shows that the ratio of the charge density of the test body to the mass density plays an important role. If it is possible to make this ratio, at least in theory, sufficiently small, then a local measurement of the field is possible. The same conclusion can be generalized for any gauge field, except for the gravitational one. In the case of gravity, the role of charge is played by gravitational mass. And according to the principle of equivalence, gravitational mass is proportional to inertial one, and their ratio remains constant. The second problem is related to the existence of a gravitational radius for any gravitating body. And since the size of the test body cannot be less than its gravitational radius, this also excludes the local measurability of the gravitational field in the quantum case.

Finally, another reason that does not allow constructing a quantum theory of gravity is the problem of defining vacuum in general relativity. Einstein's equations in the absence of matter have three wellknown solutions: de Sitter worlds of the 1st and 2nd kind, as well as the Minkowski world. On the other hand, from the constancy of the speed of light in a vacuum, it follows that the laws of physics in the absence of matter are invariant not only concerning the Lorentz group but also concerning the conformal group $\mathrm{SO}(4 ; 2)$. Moreover, H.Weyl even constructed a conformal invariant theory of gravity, [8]. For the vacuum problem, we are interested in the fact that all three groups - de Sitter groups of the 1st and 2nd kind $S O(4,1)$ and $S O(3,2)$, respectively, as well as the Poincare group, are subgroups conformal group $S O(4,2)$, /see Appendix/4.

All this shows that the well-known methods of quantizing gauge fields, in particular the electromagnetic field, are inapplicable to the gravitational field. It is usually said that the gravitational field is not quantized. However, a different conclusion can and should be drawn. Recall that as a result of the unification of the special theory of relativity with quantum mechanics, a new object appears - a relativistic quantum field. This one is the basic concept of high-energy physics. But from the above, it follows that the result of combining general relativity with quantum mechanics will not be a quantized field, but a new object. From a mathematical point of view, the essence of the problem is that in the general theory of relativity, coordinates have neither metric nor physical meaning. They have only topological functions. Therefore, we must abandon standard methods and develop new ones and concepts that are adequate for general relativity. In our research, we will be based on the properties of the Einstein equations and their solutions. We have to reformulate the concepts in terms of solutions to Einstein's equations. Thus, we can achieve a unification of general relativity and quantum mechanics and obtain concepts that can replace analogs of the elementary system and Wigner's theorem in terms of solutions to Einstein's equations.

\footnotetext{
${ }^{4}$ In our opinion, the interesting work [25] allows for a different interpretation.
} 
This program is the basis for our further research.

\section{APPENDIX}

The group $S O(4,2)$ is a connected component of the unit of the group of motions of the 6-dimensional pseudo-Euclidean space, which leaves the quadratic form invariant, $[14,19]$ :

$$
[k, k]=k_{1}^{2}+k_{2}^{2}+k_{3}^{2}+k_{4}^{2}-k_{5}^{2}-k_{6}^{2}
$$

Elements of $g \in S O(4,2)$ are represented by $6 \times 6$ - matrices that perform linear transformations over $k=\left(k_{1}, k_{2}, k_{3}, k_{4}, k_{5}, k_{6}\right)$. From the definition, it follows that the relations are satisfied:

$$
g^{\top} \eta g=\eta, \quad \operatorname{det}(g)=1,
$$

where the T means transposition and $\eta$ is the diagonal $6 \times 6$-matrix:

$$
\eta=\operatorname{diag}(1,1,1,1,-1,-1) .
$$

The groups $S O(4,1)$ and $S O(3,2)$ are subgroups of the conformal group $S O(4,2)$. Let us show that the Poincare group $I S O(3,1)$ is also a subgroup of $S O(4,2)$.

Transitivity surfaces of the group $S O(4,2)$ :

$$
[k, k]=k_{1}^{2}+k_{2}^{2}+k_{3}^{2}+k_{4}^{2}-k_{5}^{2}-k_{6}^{2}=c, \quad c \in \mathbb{R}
$$

pass into themselves under transformations from the group $S O(4,2)$. Depending on the sign of $c$, there are three types of transitive surfaces: a hyperboloid, if $c>0$ or $c<0$, and a cone with a punctured vertex, if $c=0$, because the point $k_{1}=k_{2}=k_{3}=k_{4}=k_{5}=k_{6}=0$ is the vertex of the cone and forms a homogeneous space.

A cone with a punctured vertex plays an exceptional role in our study, so we restrict ourselves to the study of invariant measures and coordinate systems on the cone.

We introduce a spherical coordinate system on the cone $[k, k]=0$ :

$$
k=\omega \boldsymbol{n}, \quad \boldsymbol{n}=(\xi, \zeta), \quad 0<\omega<\infty, \quad[\boldsymbol{n}, \boldsymbol{n}]=0 .
$$

Here $\xi$ and $\zeta$ are 4-dimensional and 2-dimensional unit vectors, respectively: ${ }^{5}$

$$
\begin{gathered}
\zeta=(\sin \psi, \cos \psi), \quad \zeta^{2}=1, \quad 0 \leq \psi<2 \pi ; \\
\xi=(\cos \chi, \sin \chi \cos \vartheta, \sin \chi \sin \vartheta \cos \varphi, \sin \chi \sin \vartheta \sin \varphi), \quad \xi^{2}=1 ; \\
0 \leq \psi<2 \pi ; \quad 0 \leq \chi, \vartheta \leq \pi .
\end{gathered}
$$

As a fixed point, $\dot{k}$ it is convenient to choose point with spherical coordinates:

\footnotetext{
${ }^{5}$ The spherical coordinate system does not constitute an atlas. Therefore, all of the following relations hold almost everywhere or for almost all elements of the group.
} 


$$
\dot{k}=(1,0,0,0,0,1) \text {. }
$$

The transformation $h(k)$ (the so-called "boost" or the Wigner operator) that maps a fixed point $\dot{k}$ to a given point $k$ is defined as follows:

$$
h(k)=g_{34}(\varphi) g_{23}(\vartheta) g_{12}(\chi) g_{56}(\psi) g_{16}(\beta), \quad \beta=\ln \omega
$$

The stationary group $W$ of the point $k$ is isomorphic to the group of motions of the 4-dimensional pseudoEuclidean space $M_{4}$, that is, Poincare group $I S O(3,1)$ :

$$
W=I S O(3,1)
$$

Each element of $w \in W$ uniquely represented as:

$$
w=R(r) B(\vec{z}),
$$

where $\vec{z}$ is a 4-dimensional vector, and $r$ is the pseudo-orthogonal $6 \times 6$-matrix:

$$
g^{\top} \hat{\eta} g=\hat{\eta}, \quad \hat{\eta}=\operatorname{diag}(1,1,1,-1) .
$$

The matrices $R(r)$ and $B(\vec{z})$ have the following form:

$$
\begin{gathered}
R(r)=\left(\begin{array}{ccc}
1 & \overrightarrow{0}^{\top} & 0 \\
\overrightarrow{0} & r & \overrightarrow{0} \\
0 & \overrightarrow{0}^{\top} & 1
\end{array}\right), \quad R(r) \in S O(3,1) \subset S O(4,2), \\
B(\vec{z})=\left(\begin{array}{ccc}
1+\frac{z^{2}}{2} & \vec{z}^{\top} & -\frac{z^{2}}{2} \\
\vec{z} & \hat{\eta} & -\vec{z} \\
\frac{z^{2}}{2} & \vec{z}^{\top} & 1-\frac{z^{2}}{2}
\end{array}\right), \quad z^{2}=\vec{z}^{2}=\vec{z}^{\top} \hat{\eta} \vec{z}=z_{1}^{2}+z_{2}^{2}+z_{3}^{2}-z_{4}^{2} .
\end{gathered}
$$

The matrices $B(\vec{z})$ form an abelian subgroup, which is a normal divisor of the stationary subgroup $\cong$ ISO $(3,1)$ :

$$
B\left(\vec{z}_{1}\right) B\left(\vec{z}_{2}\right)=B\left(\vec{z}_{1}+\vec{z}_{2}\right), \quad R\left(r^{-1}\right) B(\vec{z}) R(r)=B\left(r^{-1} \vec{z}\right) .
$$

Thus, for $g \in S O(4,2)$ we have:

$$
g=h(k) w
$$

Using the formulas (19)-(20), we obtain the expansion for the elements of the group $(4,2)$ :

$$
g=g_{34}(\varphi) g_{23}(\vartheta) g_{12}(\chi) g_{56}(\psi) g_{16}(\beta) R(r) B(\vec{z})
$$

Thus, the groups $S O(4,1), S O(3,2)$, and $I S O(3,1)$ are, up to isomorphism, subgroups of the conformal group $S O(4,2)$. 


\section{REFERENCES}

[1] Ryabov VA, Tsarev VA, Tskhovrebov AM. The Search for Dark Matter Particles, Uspekhi Fizicheskikh Nauk 178 (11) 1129 - 1163 (2008) (in Russian).

[2] New Ideas in Dark Matter 2017, arXiv: 1707.0459v1 [hep-oh], 2017, p.113.

[3] Weinberg S. The Quantum Theory of Fields, vol.1-2, Cambridge University Press, 1995-1998.

[4] Weinberg S. Gravitation and Cosmology, John Wiley and Sons, Inc., New York-LondonSydney-Toronto, 1972

[5] Einstein A, Collected Works, vols. 1-4, "Nauka", Moscow, 1965-1967 (in Russian).

[6] Hawking S and Ellis G. The Large Scale Structure of Space-Time, Cambridge University Press, 1973

[7] Mustapha Ishak Testing General Relativity in Cosmology, arXiv:1806.10122v1 [astro-ph.CO] 26 Jun 2018.

[8] W.Pauli, Theory of Relativity, Pergamon Press, 1958.

[9] Wigner E. On Unitary Representations of the Inhomogeneous Lorentz Group, Nuclear Physics B (Proc.Suppl.), 6, (1989), 9-64; Ann. Math., 40, 149 (1939).

[10] Exact Solutions of the Einstein's Field Equations, ed. by E.Schmutzer, Deutscher Verlag der Wissenschaften, Berlin, 1980.

[11] Groen O, Hervik S. Einstein's General Theory of Relativity (book draft, 2004), 538s.

[12] Galtsov DV, Particles and fields near black holes, Moscow University Press, 1986 (in Russian).

[13] Wigner, Inönü, Proc. Nat. Acad. Sci.(USA), 39, 510, 1953; 40, 119 (1954).

[14] Barut AO, Raczka R, Theory of Group Representations and Applications, Warsaw, 1977.

[15] Bohr N. Über die Anwendung Quantentheorie auf den Atombau. I. Grundpostulate der Quantentheorie, Zs.f.Phys., 1923, 13, 117-165.

[16] Bohr N, Selected Works, vols 1-2, Moscow, "Nauka", 1970-1971 (in Russian).

[17] Weinberg S. Lectures on Quantum Mechanics, Cambridge University Press, 2013.

[18] Messiah A, Quantum Mechanics, vols I-II, North Holland, John Wiley \& Sons, 1966.

[19] Bargmann V. On Unitary Ray Representations of Continuous Groups - Ann. of Math., 1954, v.59, No.1, p.1-46.

[20] Gürsey F, Introduction to Group Theory, in "Relativity, Groups and Topology”, eds. C. De-Witt, B. DeWitt, New York-London, 1964. 
Rajabov / Eskişehir Technical Univ. J. of Sci. and Tech. A-Appl. Sci. and Eng. Vol. 21-2020

[20] Rajabov BA. Trans. Nat. Acad. Sci., Azerbaijan, Series of Phys.-Math. Sciences, 1983; No.6, pp.58-63; (in Russian).

[21] Rajabov BA. The Contraction of the Representations of the Group $\mathrm{SO}(4,1)$ and Cosmological Interpretation, Astronomy \& Astrophysics (Caucasus), 3, (2018), pp.74-90; arXiv:1801.10004, p.12.

[22] Monin AS, P.Ya. Poloubarinova-Kochina, V.I. Khlebnikov, Cosmology, Hydrodynamics, Turbulence - "Nauka", Moscow, 1989 (in Russian).

[23] General Relativity, ed. S.W. Hawking, W. Israel - Cambridge University Press, 1979.

[24] Bohr N. Rosenfeld L. Field and Charge Measurements in Quantum Electrodynamics, Phys.Rev., 1950, 78, 794-798.

[25] Senarath P. de Alwis, Francesco Muia, Veronica Pasquarella and Fernando Quevedo, Quantum Transitions Between Minkowski and de Sitter Spacetimes, arXiv:1909.01975v1 [hep-th] 4 Sep 2019. 